\title{
(c)
}

(c) Auteur. Cette œuvre, disponible à

http://dx.doi.org/10.18162/fp.2021.a225, est distribuée

sous licence Creative Commons Attribution 4.0 International

http://creativecommons.org/licences/by/4.0/deed.fr

Mathieu Thibault

Université du Québec en Outaouais (Canada)

\section{COVID-19: COurs avec VIDéo - 19 conseils}

doi: $10.18162 / f p .2021$. .a225

\section{HRONIQUE • Technologies en éducation}

Pour organiser la structure de cette réflexion pédagogique, j’ai pensé que COVID-19 pouvait aussi vouloir dire COurs avec VIDéo - 19 conseils. Quelques études empiriques suggèrent que l'enseignement universitaire à l'aide de capsules vidéo aurait un impact sur la performance académique dans des domaines variés (voir par exemple, Expósito et al., 2020; Pereira et al., 2004). Toutefois, d'autres études font ressortir que le recours aux capsules n'influence pas nécessairement la performance des étudiants universitaires et certaines soulèvent parfois des inconvénients (voir par exemple, Smith et al., 2012). Devant de tels résultats contradictoires, un jugement critique est toujours de mise. Selon Karsenti et Collin (2011), les capsules offrent un apport significatif à la formation universitaire à distance en enseignement. Parmi les apports de l'enseignement-apprentissage à l'aide de capsules, il convient de souligner la possibilité d'accroître la motivation et l'engagement des étudiants (Tiernan, 2015), de favoriser un apprentissage flexible et asynchrone qui respecte leur rythme et leur disponibilité, puis d'exploiter le mobile learning. Ce mode d'enseignement comporte aussi des limites, notamment une perte de soutien personnalisé et de contact humain, en plus des défis techniques et d'accessibilité des outils technologiques (Mardis, 2009). Pour favoriser l'enseignement universitaire à distance à l'aide de capsules, je partage dix-neuf conseils simples, répartis dans les phases de préparation, production, puis diffusion. 


\section{Phase de préparation}

\section{1 - Envisagez la place que vous voulez accorder aux capsules vidéo dans votre cours}

D'abord, il semble important de se demander si les capsules viseront un soutien ponctuel ou si elles viseront plutôt à organiser le cours au complet. Puisque le temps de préparation des capsules est assez long, le choix des contenus à enseigner sous cette forme devient alors particulièrement important (O’Flaherty et Phillips, 2015).

\section{2 - Commencez par utiliser ce qui est déjà créé}

Si possible, je vous suggère d'exploiter des capsules existantes pour ne pas avoir à réinventer la roue. Vous pourrez constater la grande diversité de capsules déjà disponibles en ligne. Pour produire de nouvelles capsules qui répondent à vos besoins particuliers, il convient d'exploiter les ressources que vous avez déjà (par exemple vos diaporamas, fichiers de traitement de texte ou documents papier), puis de les adapter pour favoriser un apprentissage à distance autonome.

\section{3 - Ciblez les caractéristiques pour chaque capsule vidéo}

Pour chaque capsule que vous envisagez de produire, les questions suivantes pourraient vous aider à bien cibler les caractéristiques de votre contexte particulier :

- Pourquoi? Quels sont les objectifs à atteindre pour votre cours?

- Pour qui? Quelles sont les caractéristiques des étudiants?

- Pour quand? À quel moment sera utilisée la capsule?

- Quoi? Quels contenus sont ciblés?

- Comment? Quels images, photos, graphiques ou animations voulez-vous utiliser?

\section{4 - Préparez les documents, en organisant le contenu de façon pertinente, cohérente et intéressante}

Je vous suggère d'élaborer votre contenu et support visuel en vous assurant de préparer des exemples variés et concrets, puis d'attirer l'attention sur les éléments importants. De plus, il s'avère important de segmenter les contenus à aborder (Fiorella et Mayer, 2018) et de planifier la complémentarité des capsules, puisque «la mise en scène de l'organisation et la hiérarchisation des capsules vidéo va induire le cheminement mental à suivre pour rentrer dans le contenu de la formation» (Duvillard, 2017, p. 2).

\section{5 - Prévoyez des documents d'accompagnement}

Vous pouvez varier les ressources offertes en intégrant vos capsules dans une séquence logique et complète de documents d'apprentissage qui favorisent l'alignement pédagogique (Biggs, 2014). En ce sens, des documents d'accompagnement tels que des quiz (par exemple, avec GoogleForms, Moodle ou EdPuzzle) et des lectures peuvent bonifier l'apprentissage. Vous pouvez aussi demander un rapport réflexif de la capsule ou un projet à partir de son contenu.

2 - Formation et profession 29(1), 2021 


\section{CHRONIOUE}

\section{6-Choisissez une façon simple d'enregistrer}

Pour enregistrer ma capsule, la vidéocapture d'écran moffre la possibilité d'enregistrer mes explications sur n'importe quel document produit par mon ordinateur (notamment un diaporama). J'utilise Loom qui est simple et gratuit (même la version Pro, pour les enseignants), mais il existe aussi de nombreuses autres options d'enregistrement (QuickTime Player, Powerpoint, Keynote, Screencast-o-matic, Camtasia, etc.). Puisque ce n'est pas la quantité d'outils qui importe, mais plutôt l'usage qu'on en fait, je vous suggère d'expérimenter un de ces outils et d'y avoir recours s'il répond à vos besoins (sans expérimenter tous les autres outils disponibles).

\section{7-Prévoyez un environnement favorable à l'apprentissage}

La qualité audio est primordiale dans une capsule, alors il est préférable de choisir un endroit calme pour l'enregistrement. De plus, si vous devez investir dans du matériel, la priorité serait d'acheter un bon microphone, particulièrement si vous souhaitez faire une captation avec une caméra et que vous vous trouvez à distance de la caméra.

\section{Phase de production}

\section{8 - Faites un test d'enregistrement et lancez-vous}

Avant d'enregistrer votre toute première capsule, il est préférable d'apprivoiser l'outil d'enregistrement choisi. Au début, je vous suggère de vous exercer en vous chronométrant, à la fois pour évaluer la durée de votre capsule et pour développer l'aisance nécessaire lorsque viendra le temps de vous enregistrer.

\section{9-Soyez concis}

Dès le début de votre capsule, je vous suggère d'annoncer les objectifs planifiés et de vous en tenir à ces objectifs. Puisque la durée de l'attention optimale sur le contenu dans une capsule se limite à environ six minutes selon Guo, Kim et Robin (2014), il vaut mieux être le plus bref possible. En ce sens, je suggère que votre capsule ne dépasse pas une durée de quinze minutes, afin d'éviter la surcharge cognitive.

\section{0 - Ajoutez de l'interactivité}

Pour favoriser un apprentissage actif, vous pouvez suggérer de mettre en pause une capsule pour permettre aux étudiants de répondre à une question ou d'accomplir une tâche. Une rétroaction sous forme de quiz permet d'évaluer la compréhension des étudiants, que ce soit de façon formative ou sommative. Pour les encourager à interagir entre eux sur le contenu d'une capsule, un forum de discussion ou un débat en classe virtuelle peut aussi s'avérer utile. 


\section{1 - Faites ressortir une synthèse}

Vers la fin de la capsule, vous pouvez demander aux étudiants ce qu'ils ont retenu, en les encourageant à mettre en pause pour noter les éléments importants sous forme d'une liste ou encore d'une carte conceptuelle (par exemple, avec Mindmeister ou Cmap). En guise de conclusion de votre capsule, vous pouvez alors présenter votre synthèse (en lien avec les objectifs précisés au début), pour enrichir la leur.

\section{2 - Ayez un ton de voix stimulant}

Pour préserver autant que possible l'attention et l'intérêt des étudiants, il est préférable d'utiliser un ton personnel, naturel, enthousiaste et dynamique pour susciter l'intérêt des étudiants (Guo, Kim et Robin, 2014).

\section{3 - Trouvez un rythme adéquat}

Selon Guo, Kim et Robin (2014), l'intérêt et l'engagement augmentent lorsque le débit de parole augmente. Toutefois, le rythme ne doit pas être trop rapide non plus et doit être adapté aux objectifs d'apprentissage.

\section{4 - Restez simple}

Certains enseignants produisent des capsules sensationnelles sur le plan visuel, mais je vous suggère de rester dans la simplicité et de favoriser la qualité du contenu plutôt que la qualité du contenant. Dans cette optique, le temps que vous pourrez investir permettra de couvrir plusieurs concepts du cours dans plusieurs capsules, plutôt qu'un nombre restreint avec davantage d'artifices visuels par des montages complexes.

\section{5 - Acceptez l'imperfection}

Je vous conseille de ne pas reprendre une capsule, même si elle n'est (évidemment) pas parfaite. Il est parfois tentant de vouloir reprendre une capsule lorsqu'on bafouille au bout de quelques minutes. Pourtant, dans une séance de cours en présentiel, on n'envisage jamais de recommencer la séance si on bafouille à un moment. Dans ce cas, on se reprend et on continue... et c'est exactement ce que je vous conseille de faire dans une capsule. Il convient de rappeler que le but est d'accompagner les étudiants dans leur apprentissage et non pas de viser à remporter un Oscar.

\section{6 - Faites concevoir une capsule vidéo par les étudiants}

Après avoir donné l'exemple aux étudiants par votre production de capsules, vous pouvez à votre tour les inviter à en concevoir une. J'apprécie l'idée de cibler des concepts du cours et d'en attribuer un par étudiant (ou par équipe), qui doit s'approprier ce concept, puis produire une capsule afin de l'expliquer clairement et succinctement aux pairs. En guise d'évaluation, la capsule devient alors un excellent moyen pour les étudiants de démontrer leurs compétences transversales, notamment l'esprit de synthèse, la créativité, et la communication orale. 


\section{CHRONIOUE}

\section{Phase de diffusion}

\section{7 - Choisissez une façon simple d'héberger votre capsule vidéo}

Pour déposer des capsules dont les fichiers occupent des tailles considérables, il est préférable d'utiliser les services de diffusion comme YouTube (ou encore Vimeo) qui se spécialisent dans ce type d'hébergement que les étudiants connaissent bien (Moghavvemi et al., 2018).

\section{8 - Partagez le lien avec les étudiants}

Il suffit alors de partager le lien de votre capsule avec les étudiants, sur votre environnement numérique d'apprentissage ou par courriel.

\section{9 - Partagez votre expérience avec vos collègues}

N'hésitez pas à partager vos bons coups et vos échecs en échangeant avec vos collègues, pour prendre un pas de recul sur votre démarche et bénéficier des idées de vos pairs. Si possible, je vous suggère de travailler en équipe pour obtenir la rétroaction d'un collègue et vous répartir la tâche de conception de capsules.

\section{Remarques conclusives}

En guise de synthèse, la figure ci-dessous énumère 19 conseils pour favoriser l'enseignement universitaire à distance à l'aide de capsules vidéo.

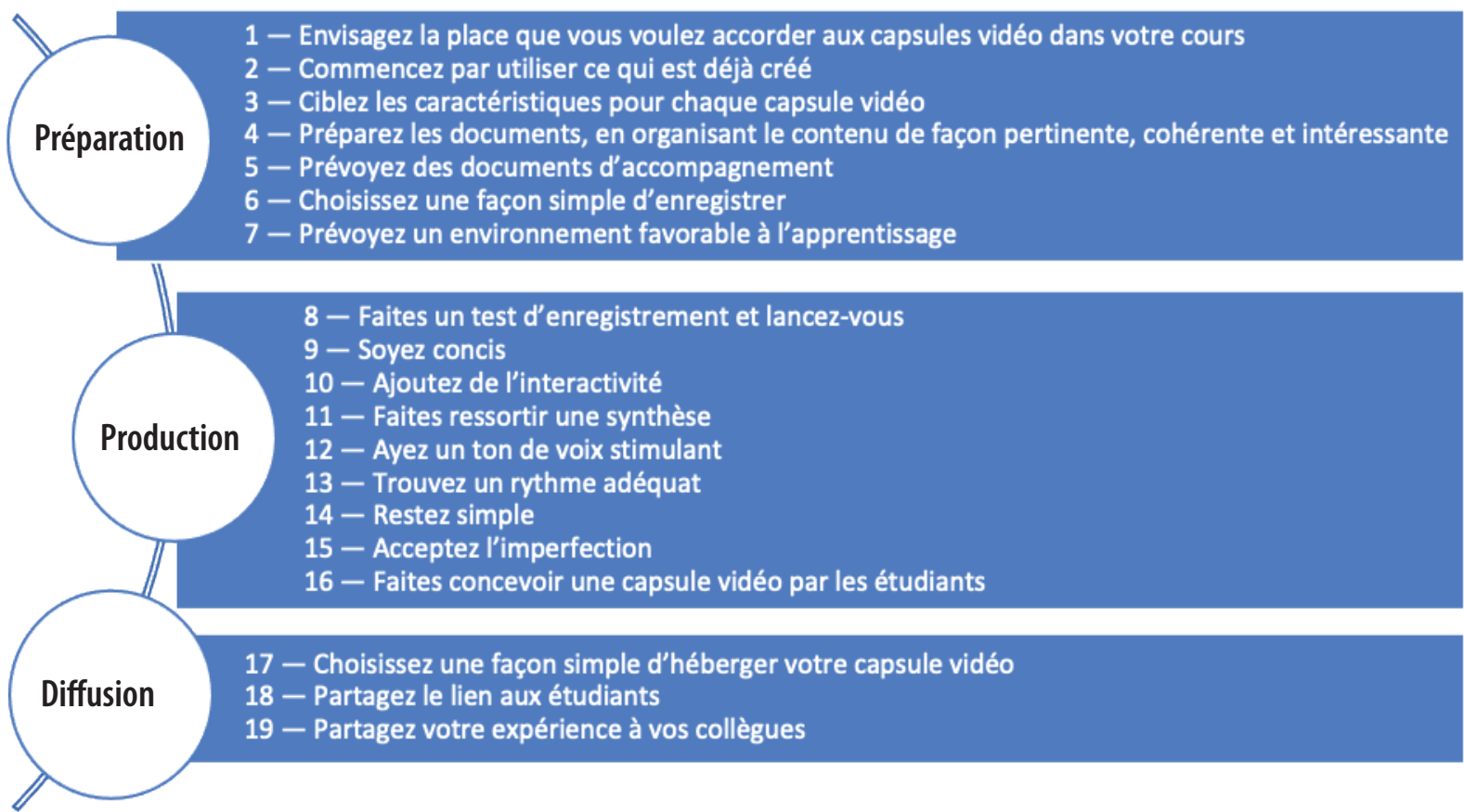


En espérant que ces conseils vous seront utiles, je vous offre un dernier conseil (en bonus) : acceptez que le changement soit déstabilisant et chronophage au début, étant donné que sortir de notre zone de confort permet parfois de faire de bien belles découvertes, au profit des étudiants!

\section{Références}

Biggs, J. (2014). Constructive alignment in university teaching. HERDSA Review of Higher Education, 1(1), 5-22. https://www.herdsa.org.au/herdsa-review-higher-education-vol-1/5-22

Duvillard, J. (2017). La capsule vidéo : un complément de formation des enseignants? Distances et médiations des savoirs, 19, 1-7.https://doi.org/10.4000/dms. 1903

Expósito, A., Sánchez-Rivas, J., Gómez-Calero, M. P. et Pablo-Romero, M. P. (2020). Examining the use of instructional video clips for teaching macroeconomics. Computers E Education, 144, 103709. https://doi.org/10.1016/ j.compedu.2019.103709

Fiorella, L. et Mayer, R. E. (2018). What works and doesn't work with instructional video. Computers in Human Behavior, 89, 465-470. https://doi.org/10.1016/j.chb.2018.07.015

Guo, P.J., Kim, J. et Robin, R. (2014). How video production affects student engagement: An empirical study of MOOC videos. Dans Proceedings of the first ACM conference on Learning@scale conference (p. 41-50). http://doi.org/10.1145/2556 $\underline{325.2566239}$

Karsenti, T. et Collin, S. (2011). The impact of online teaching videos on Canadian pre-service teachers. Campus-Wide Information Systems, 28(3), 195-204. https://doi.org/10.1108/10650741111145724

Mardis, M.A. (2009). Viewing Michigan's digital future : results of a survey of educators' use of digital video in the USA, Learning, Media and Technology, 34(3), 243-257. https://doi.org/10.1080/17439880903141539

Moghavvemi, S., Sulaiman, A., Jaafar, N. I., \& Kasem, N. (2018). Social media as a complementary learning tool for teaching and learning: The case of youtube. International Journal of Management in Education, 16, 37-42. https://doi. org/10.1016/j.ijme.2017.12.001

O'Flaherty, J. et Phillips, C. (2015). The use of flipped classrooms in higher education: A scoping review. The internet and higher education, 25, 85-95. https://doi.org/10.1016/j.iheduc.2015.02.002

Pereira, J.A., Meri, A., Masdeu, C., Molina-Tomas, M.C. et Martinez-Carrio, A. (2004). Using videoclips to improve theoretical anatomy teaching. Eur J Anat, 8(3), 143-146. http://eurjanat.com/data/pdf/eja.04030143.pdf

Smith, W., Rafeek, R., Marchan, S. et Paryag, A. (2012). The use of video clips as a teaching aide. European Journal of Dental Education, 16(2), 91-96. https://doi.org/10.1111/j.1600-0579.2011.00724.x

Tiernan, P. (2015). An inquiry into the current and future uses of digital video in University teaching. Educ Inf Technol, 20, 75-90. https://doi.org/10.1007/s10639-013-9266-8

\section{Pour citer cet article}

Thibault, M. (2021). COVID-19 : Cours avec VIDéo - 19 conseils [chronique]. Formation et profession, 29(1), 1-6. http://dx.doi.org/10.18162/fp.2021.a225 